\title{
Judging the time to collision with a simulated textured object: Effect of mismatching rate of expansion of object size and of texture element size
}

\author{
ALEX VINCENT and DAVID REGAN \\ York University, North York, Ontario, Canada
}

\begin{abstract}
We measured the accuracy with which subjects estimated the time to collision with a simulated textured object approaching at constant speed along the line of sight. The independent variable was the ratio $R$, where $R=$ (rate of dilation of the texture elements that covered the simulated object)/ (rate of dilation of object size). When matching was perfect (i.e., $R=1.0$ ), the mean of 12 settings was close to the nominal value of 2,000 msec for both subjects. In addition, the standard error of 12 settings was only 25 and $52 \mathrm{msec}$ in $2,000 \mathrm{msec}$ for the 2 subjects. Discrimination threshold for time to collision was not significantly affected by $R$ over the range investigated between $R=0$ and $R=2.0$. However, the accuracy of estimating time to collision was significantly affected by $R$. Estimated time to collision was a monotonic function of $R$. For example, when the mismatch was only $10 \%$ (i.e., $R=0.9$ ) subjects judged time to collision would occur $178 \mathrm{msec}$ later than the true time to collision of $2,000 \mathrm{msec}$.
\end{abstract}

A retinal image correlate of time to collision $(T)$ with an approaching object is given by

$$
T \approx \frac{\theta}{d \theta / d t}
$$

where $\theta$ is the object's angular subtense at the observer's eye (Hoyle, 1957). ${ }^{1}$ Following Lee (1976), it has been suggested that humans use Equation 1 to help them make judgments of time to collision in sporting activities, highway driving, and aviation - though it has been pointed out that a binocular correlate of time to collision is also available and can be used, and, furthermore, that alternative visual control strategies could get the observer's hand (or foot) to the right location at the right time without explicitly recovering time to collision (Bootsma \& van Wieringen, 1990; Cavallo \& Laurent, 1988; Gray \& Regan, 1996a, 1996b; Karnavas, Bahill, \& Regan, 1990; Kruk \& Regan, 1983, 1996; Lee \& Lishman, 1977; Lee, Lishman, \& Thomson, 1982; Lee, Young, Reddish, Lough, \& Clayton, 1983; McBeath, Shaffer, \& Kaiser, 1995; McLeod \& Dienes, 1993; Peper, Bootsma, Mestre, \& Bakker, 1994; Regan, 1991, 1992, in press; Savelsbergh, Whiting, \& Bootsma, 1991; Schiff \& Detwiler, 1979; Todd, 1981; Warren, Young, \& Lee, 1986).

This work was sponsored by the U.S. Air Force Office of Scientific Research, Air Force Systems Command (Grant F49620-94-1-0083 to D.R.). The U.S. government is authorized to reproduce and distribute reprints for governmental purposes notwithstanding any copyright violation thereon. A.V. was supported by the Institute for Space and Terrestrial Science, ON. D.R. holds the CAE/NSERC Industrial Research Chair of Vision in Aviation. Correspondence should be addressed to D. Regan, York University, BSB Room 375, 4700 Keele St., North York, ON Canada M3J 1P3, (e-mail: martin@george.psych.yorku.ca).
The accuracy with which some sport players can time a hit is remarkable-from \pm 2 to $\pm 3 \mathrm{msec}$ accuracy for top-class cricket players and national-level table tennis players (Bootsma \& van Wieringen, 1990; Regan, 1992; Regan, Beverley, \& Cynader, 1979). In these real-world situations, the approaching object is usually textured, and its retinal image obeys the laws of projective geometry: In particular, the rate of expansion of the object's retinal image size exactly matches the rate of expansion of the texture elements within the object's retinal image. However, in the visual simulation and virtual reality literature, simulated terrain features were not textured and remained cartoon-like in even the most advanced flight simulators until the late 1970s. Since that time, however, technical advances in computers and virtual reality display technology have allowed greater and greater scene content to be introduced, including texturing of terrain features.

In the present study, we investigated what effect simulated surface texture has on judging time to collision with a simulated object. Our rationale is as follows. The aim of a previous study was to measure the effectiveness of textured approaching objects as stimuli for motion-in-depth perception (Beverley \& Regan, 1983). There were nine textured test squares and one untextured test square, all of which had the same starting retinal image size and rate of dilation of size, but with different rates of dilation of the texture elements. Because we found it difficult to attend exclusively to the motion-in-depth component of the complex percept produced by some of this stimulus set, we used an indirect method for comparing their effectiveness as stimuli for motion-in-depth perception. This method was to measure the speed of the motion-indepth aftereffect produced by separately adapting to each 
one of the test squares. Aftereffect speed was measured by cancellation, and the canceling target was the same in every case: an untextured, uniformly illuminated square with the same starting size as the textured test squares. The main finding of this study was that a textured test square that had the same rate of expansion of square size and texture element size produced only a slightly faster motion-in-depth aftereffect than an untextured test square, but considerably slower aftereffects were produced by textured test squares whose rate of expansion of texture element size was less than the rate of expansion of square size. The conclusion was that in flight simulator or other virtual reality displays, a failure to match the rates of expansion of the size of simulated textured objects to the rate of expansion of texture element size might degrade the effectiveness of simulator training. ${ }^{2}$ Below we report a laboratory investigation on the effect of mismatches on judgments of time to collision.

\section{METHOD}

\section{Simulation of Motion in Depth}

When a rigid, nonrotating object moves directly toward the eye at constant speed, providing $\theta_{0}$ and $\theta_{t}$ are small, the angular size of the object's retinal image increases according to the equation

$$
\theta_{t} \approx \frac{\theta_{0}}{\left(1-t / T_{0}\right)},
$$

where $\theta_{0}$ and $T_{0}$ are, respectively, the angular size of the object's retinal image and the time to collision at time $t=0$ and $\theta_{t}$ is the angular size of the object's retinal image at time $t$ (Regan \& Hamstra, 1993). In the present study we used an IBM PC to load the segment of Equation 2 between $t=0$ and $t=0.67 T_{0}$ into the memory of an arbitrary waveform generator (Wavetek Model 75). The generator had a resolution of 1 part in 2,048 on the $y$ (voltage) -axis and 1 part in 8,192 on the $x$ (time) -axis. The $x$-axis was clocked by a signal generator whose frequency was monitored to within $0.1 \mathrm{~Hz}$ (i.e., $0.0016 \%$ for a 2,000 -msec time to collision) by a digital frequency meter (Fluke, Model 1900A).

\section{Apparatus}

A textured square was generated by hardware electronics of our own design (Beverley \& Regan, 1983) and displayed on an electrostatically driven monitor (Tektronix Model 608) at a frame rate of $49 \mathrm{~Hz}$. The luminance of the bright elements was $33 \mathrm{~cd} / \mathrm{m}^{2}$ and the contrast was nearly $100 \%$. The textured square was optically superimposed on a uniformly illuminated disk subtending $9.7^{\circ}$ and of luminance $18 \mathrm{~cd} / \mathrm{m}^{2}$, so that the monitor itself could not be seen. Subjects viewed the display from a distance of $70 \mathrm{~cm}$. One eye was occluded.

The starting size of the textured square was always $1.2^{\circ}$ and the largest finishing square size (at the end of the longest presentation duration) was $4.1^{\circ}$. The voltage output of the arbitrary waveform generator controlled the size of the textured square according to Equation 2. Thus, the angular size of the textured square in Figure 1 increased with the same time course as the image of a square object approaching the eye at constant speed. The simulated time to collision at time $t=0$ was nominally $2,000 \mathrm{msec}$, and this was held constant throughout the experiment.

The angular size of any given texture element was changed with time according to Equation 2 so as to simulate a constant-speed approach. The time to collision with the texture elements was equal to $2,000 / R$. Only when $R=1.0$ was the time to collision for any given texture element the same as the fixed $2,000-\mathrm{msec}$ time to collision for the square. For $R>1.0$, the texture elements would arrive before the square object and for $R<1.0$, the texture elements
A
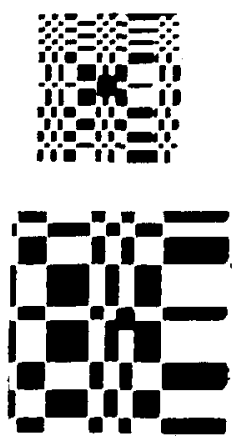
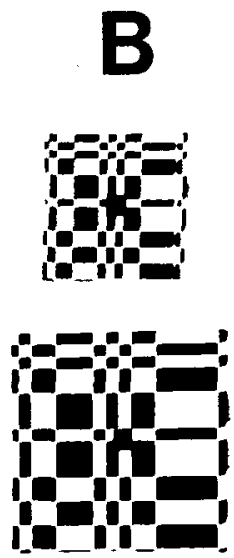

C
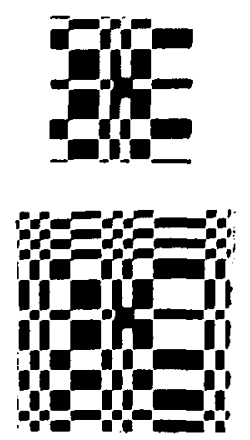

Figure 1. Photographs of three of the stimulus displays. The conditions shown are $R=2.0, R=1.0$, and $R=0.0$ in $A, B$, and $C$, respectively, where $R=$ (rate of dilation of the texture elements within the square)/(the rate of dilation of the square).

would arrive after the square object. We varied $R$ between 2.0 (Figure $1 \mathrm{~A}$ ) through 1.0 (Figure $1 \mathrm{~B}$ ) to 0.0 (Figure $1 \mathrm{C}$, static texture). ${ }^{3}$

\section{Calibration Errors}

The nominal value of time to contact of $2,000 \mathrm{msec}$ was not, of course, realized with zero error. Compared with the error associated with the calibration to the size of the stimulus square, we can neglect the errors in both the clock rate of the arbitrary waveform generator and the voltage delivered by the arbitrary waveform generator. To estimate the calibration error, we fitted a regression line to a plot of the square's side length versus the voltage applied to the square generator electronics, and calculated the standard error of the regression line. From Equation 1 we then estimated a standard error of $32 \mathrm{msec}$ in the $2,000-\mathrm{msec}$ nominal value of time to collision.

\section{Psychophysical Procedure and Data Analysis}

Data were collected using a transformed up-down staircase procedure (Levitt, 1971). The sequence was started when the subject pressed a button. Following a pretrial interval of $2.5 \mathrm{sec}$, the expanding textured square appeared on the monitor with a side length of $1.2^{\circ}$. The square was switched off at the end of the presentation. Some time later a brief $(50-\mathrm{msec})$ click was generated. The subject was instructed to press one of two buttons, depending on whether the simulated object would have reached his eye before or after the click.

Data were collected for a 2,000-msec time to collision for the following values of $R: 0.0,0.5,0.8,0.9,1.0,1.2$, and 2.0. Presentation duration was varied randomly from trial to trial over a range of 0.735 to $1.37 \mathrm{sec}$ (flat probability function) with a mean of $1.05 \mathrm{sec}$. Three tracking laws were interleaved randomly. They converged onto the $71 \%, 50 \%$, and $29 \%$ "sooner than the click" response probabilities, respectively (Levitt, 1971). Each of the three endpoints was estimated by calculating the mean of the last six peaks and valleys in the relevant run as described by Levitt. We labeled the locations along the time axis of the $71 \%, 50 \%$, and $29 \%$ probabilities of the "sooner than the click" response as, respectively, $T_{71}, T_{50}$, and $T_{29}$.

One reason for placing convergence points symmetrically on either side of the $50 \%$ point in addition to the $50 \%$ itself was to check that our stimulus manipulations did not render the psychometric function asymmetric about the $50 \%$ point. Such an asymmetry would have hindered the interpretation of any change in slope and also the interpretation of the location of the $50 \%$ point. We measured the accuracy of judgment by locating the $50 \%$ point. ( $T_{50} \mathrm{can}$ be regarded as a subject's estimate of time to collision.) We measured the precision of judgment in terms of the discrimination threshold $\Delta T$, defined as $\Delta T=0.5\left(T_{29}-T_{71}\right)$. 


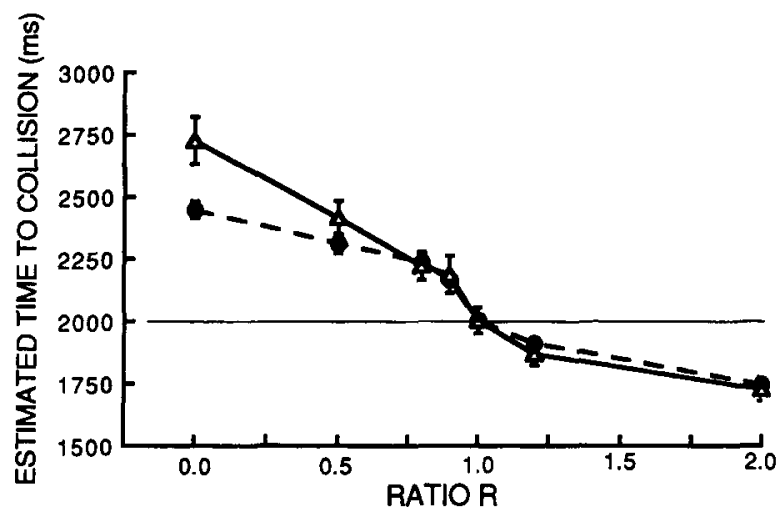

Figure 2. Estimated time to collision in milliseconds is plotted as ordinate versus the ratio $R$, where $R=$ (rate of dilation of the texture elements within the square)/(rate of dilation of the square). Filled circles are for Subject 1 and open triangles are for Subject 2 . The vertical bars indicate \pm 1 standard error.

Subjects. Two subjects were used. Subject 1 (author A.V.) was a male aged 30 . Subject 2 was a male aged 20 who was paid for his participation. Subject 2 had no previous experience in psychophysics or perception and was naive regarding the aims of the experiment. Both subjects had monocular visual acuity of $20 / 20$ or better.

\section{RESULTS}

Figure 2 shows a plot of the estimated time of collision (i.e., $T_{50}$ ) versus the ratio $R$. Solid circles (dashed line) are for Subject 1 and open triangles (continuous line) are for Subject 2. Figure 2 shows that the estimated time to collision grew progressively shorter as $R$ was increased and that the estimate was most accurate in the $R=1.0$ condition. Each data point in Figure 2 was based on 12 tracking runs. Table 1 sets out the numerical results.

We checked the symmetry of each of the individual psychometric functions by using a $t$ test to compare the values of $\left(T_{29}-T_{50}\right)$ and $\left(T_{50}-T_{71}\right)$. In none of the 14 conditions presented in Table 1 was there a significant difference, the smallest $p$ value being .38 (Table 2).

A $t$ test was used to calculate the significance of the errors in estimating time to collision, and the results are presented in Table 2 . The " $T_{50}$ versus 2,000 " column in Table 2 lists the significance levels of these errors. In the $R=1.0$ condition, the estimated time to contact $\left(T_{50}\right)$ was not significantly different from the nominal value of $2,000 \mathrm{msec}$ for Subject $1(p=.93)$ and for Subject $2(p=$ .96). For both subjects, $T_{50}$ was significantly different from $2,000 \mathrm{msec}$ for all other values of $R$.

Discrimination threshold for time to collision $(\Delta T$ in Table 1) was effectively independent of $R$ : The difference between $\Delta T$ at any given value of $R\left[(\Delta T)_{R}\right]$ and $\Delta T$ for $R=1.0\left[(\Delta T)_{1.0}\right]$ was not significant for any value of $R$, the smallest $p$ value being .2 (Table 2). The mean value of discrimination threshold over all values of $R$ was $159 \mathrm{msec}$ for Subject 1 and $178 \mathrm{msec}$ for Subject 2 . Expressed as a percentage of $2,000 \mathrm{msec}$, these thresholds were $8.0 \%$ and $8.9 \%$.

\section{DISCUSSION}

\section{Effect of Mismatching Rates}

Two possible effects of varying the ratio $(R)$ between the rates of dilation of texture element size and square size are that (1) the slope of the psychometric function might change and (2) the location of the $50 \%$ point might shift along the time axis. The first effect would correspond to a change in the discrimination threshold for time to collision, and the second to a change in the absolute accuracy of judging time to collision.

We found that varying the value of $R$ from 0.0 to 2.0 produced no significant effect on discrimination threshold for time to collision. Discrimination threshold was $8.0 \%$ for Subject 1 and $8.9 \%$ for Subject 2 . This compares with previous estimates of $10 \%, 5.5 \%, 6.7 \%$, and $9.3 \%$ for discrimination threshold obtained for 4 subjects using a different psychophysical procedure with an untextured target (Regan \& Hamstra, 1993; Regan \& Vincent, 1995).

Our main finding is that subjects make significant errors in judging time to collision when the rate of dilation of texture within the simulated approaching object differs from the rate of dilation of object size. For example, when the mismatch was only $10 \%(R=0.9)$, our subjects judged that collision would occur $178 \mathrm{msec}$ later than the

Table 1

Estimates of Time to Collision for Seven Values of $\boldsymbol{R}$

\begin{tabular}{|c|c|c|c|c|c|c|c|c|c|c|c|c|c|c|}
\hline \multirow[b]{3}{*}{ Subject } & \multicolumn{14}{|c|}{ Ratio $R$} \\
\hline & \multicolumn{2}{|c|}{0.0} & \multicolumn{2}{|c|}{0.5} & \multicolumn{2}{|c|}{0.8} & \multicolumn{2}{|c|}{0.9} & \multicolumn{2}{|c|}{1.0} & \multicolumn{2}{|c|}{1.2} & \multicolumn{2}{|c|}{2.0} \\
\hline & Est. & $S E$ & Est. & $S E$ & Est. & $S E$ & Est. & $S E$ & Est. & $S E$ & Est. & $S E$ & Est. & $S E$ \\
\hline $1 T_{50}$ & 2,449 & 35 & 2,314 & 40 & 2,239 & 33 & 2,168 & 28 & 2,004 & 25 & 1,913 & 18 & 1,746 & 28 \\
\hline$T_{71}$ & 2,318 & 49 & 2,158 & 25 & 2,102 & 25 & 1,989 & 22 & 1,861 & 32 & 1,782 & 19 & 1,633 & 24 \\
\hline$T_{29}$ & 2,643 & 51 & 2,496 & 34 & 2,402 & 37 & 2,326 & 30 & 2,197 & 42 & 2,051 & 27 & 1,907 & 29 \\
\hline$\Delta_{T}$ & 162 & 11 & 172 & 17 & 152 & 15 & 169 & 11 & 156 & 11 & 159 & 19 & 146 & 13 \\
\hline $2 T_{50}$ & 2,727 & 95 & 2,416 & 69 & 2,223 & 57 & 2,188 & 75 & 2,003 & 52 & 1,870 & 46 & 1,727 & 46 \\
\hline$T_{71}$ & 2,517 & 95 & 2,206 & 46 & 2,039 & 57 & 2,000 & 77 & 1,809 & 53 & 1,752 & 48 & 1,585 & 47 \\
\hline$T_{29}$ & 2,925 & 92 & 2,597 & 71 & 2,410 & 57 & 2,367 & 107 & 2,152 & 42 & 2,026 & 37 & 1,925 & 43 \\
\hline$\Delta_{T}$ & 199 & 26 & 175 & 24 & 185 & 18 & 185 & 24 & 164 & 15 & 155 & 15 & 182 & 22 \\
\hline
\end{tabular}

Note-The nominal value was $2,000 \pm 32 \mathrm{msec} . T_{50}, T_{71}$, and $T_{29}$ are the times of occurrence of a brief click that gave, respectivley, a $50 \%, 71 \%$, and $29 \%$ probability of judging that the collision with the simulated approaching object would occur before the click. 
Table 2

Results of $\boldsymbol{t}$ Test Used to Calculate Significance of Errors in Estimating Time to Collision

\begin{tabular}{|c|c|c|c|c|c|c|}
\hline \multirow[b]{2}{*}{ Ratio $R$} & \multicolumn{2}{|c|}{$T_{50}$ vs. 2,000} & \multicolumn{2}{|c|}{$(\Delta T)_{R}$ vs. $(\Delta T)_{1.0}$} & \multicolumn{2}{|c|}{$\left(T_{29}-T_{50}\right)$ vs. $\left(T_{50}-T_{71}\right)$} \\
\hline & $t$ & $p$ & $t$ & $p$ & $t$ & $p$ \\
\hline \multicolumn{7}{|l|}{ Subject 1} \\
\hline 0.0 & 10.5 & .0001 & 0.47 & .65 & 0.52 & .61 \\
\hline 0.5 & 6.1 & .0003 & 0.67 & .52 & 0.28 & .79 \\
\hline 0.8 & 5.2 & .0004 & 0.14 & .89 & 0.17 & .87 \\
\hline 0.9 & 3.9 & .0016 & 0.81 & .44 & 0.61 & .56 \\
\hline 1.0 & 0.10 & .93 & & & 0.48 & .64 \\
\hline 1.5 & 2.4 & .02 & 0.15 & .88 & 0.64 & .53 \\
\hline 2.0 & 6.0 & .0003 & 0.46 & .65 & 0.91 & .38 \\
\hline \multicolumn{7}{|l|}{ Subject 2} \\
\hline 0.0 & 7.2 & .0002 & 1.38 & .20 & 0.001 & .99 \\
\hline 0.5 & 5.5 & .0003 & 0.36 & .72 & 0.5 & .63 \\
\hline 0.8 & 3.4 & .003 & 1.1 & .30 & 0.21 & .83 \\
\hline 0.9 & 2.3 & .02 & 0.84 & .42 & 0.57 & .58 \\
\hline 1.0 & 0.05 & .96 & & & 0.05 & .96 \\
\hline 1.5 & 2.3 & .02 & 0.49 & .64 & 0.42 & .68 \\
\hline 2.0 & 6.4 & .0004 & 0.59 & .57 & 0.24 & .81 \\
\hline
\end{tabular}

Note-Column 1: values of the ratio $(R)$ between the rate of dilation of texture elements within the stimulus square and the rate of dilation of the square. Columns 2 and 3: test results on the difference between a subject's estimate of the time to collision $\left(T_{50} \mathrm{msec}\right.$ ) and the nominal value (i.e., $2,000 \pm 32 \mathrm{msec}$ ). Columns 4 and $5:$ test results on the difference between discrimination threshold for time to contact with $R=1.0\left[(\Delta T)_{1.0}\right]$ and discrimination threshold with $R$ not equal to $1.0\left[(\Delta T)_{R}\right]$. Columns 6 and $7:$ test results on the difference between $\left(T_{29}-T_{50}\right)$ and $\left(T_{50}-T_{71}\right)$, where $T_{29}, T_{50}$, and $T_{71}$ are, respectively, the time of occurrence of a brief click that gives a $50 \%, 71 \%$, and $29 \%$ probability of judging that the collision with the simulated approaching object would occur before the click.

nominal time to collision of $2,000 \mathrm{msec}$ (averaged over both subjects).

\section{Theoretical Implications}

Suppose that an observer views a bright, untextured isotropically expanding square whose edges have an instantaneous subtense of $\theta^{\circ}$. The well-known observation that the square appears to move in depth can be explained in terms of the following three-stage psychophysical model: The velocities of the square's four edges are signaled by four local motion detectors (modified Reichardt detectors); two pairs of local motion detectors feed two relative motion detectors that signal the difference in the velocities of opposite edges (Regan \& Beverley, 1978, 1980); the two relative motion detectors feed a motionin-depth stage that outputs a motion-in-depth signal, provided that retinal image expansion is isotropic - that is, that $\theta_{H} /\left(d \theta_{H} / d t\right)=\theta_{V} /\left(d \theta_{V} / d t\right)$, where $\theta_{H}$ and $\theta_{V}$ are, respectively, the angular width and height of the square (Beverley \& Regan, 1979, 1980). This model is illustrated in Figure 10 in Regan and Hamstra (1993). The model was based on visual responses to untextured simulated objects.

Turning to textured objects, our present findings, taken together with the effect of adapting to textured expanding objects (Beverley \& Regan, 1983, and see above), can be understood if the motion-in-depth stage compares the rate of dilation, not only of the square's horizontal and vertical edges, but also of contours over the entire extent of the square's surface. As already mentioned, a strong motionin-depth output is produced only if the rates of dilation of the square's horizontal and vertical edges are equal (Beverley \& Regan, 1979). We now conclude that this signal is weighted with the rate of dilation of contours over the surface, so that time to collision is underestimated if $R>1.0$ and overestimated if $R<1.0$. The weighting, however, is small: When the square's rate of dilation corresponded to a time to collision of $2,000 \mathrm{msec}$ and the texture's rate of dilation corresponded to $1,000 \mathrm{msec}$, observers estimated time to collision to be $1,725-1,750$ msec.

Our present findings are consistent with the suggestion that the perceived speed of motion in depth of a monocularly viewed object is determined not by the object's absolute speed in 3-dimensional space but rather by the rate of dilation of its retinal image (Regan \& Hamstra, 1993; Gray \& Regan, 1996a, 1996b).

\section{Implications for Simulation}

Flight simulators are widely used to train the pilots of commercial heavy jets, and are also used at frequent intervals to examine pilots' skills in emergency situations. Flight simulators can be regarded as being at the upper end of virtual reality technology, as can the highwaydriving simulators used by automobile designers and the ship-docking simulators used to train ship's captains.

An important component of flight simulator training is the learning of fast and appropriate motor responses to events in the simulated visual environment. For example, the ability to judge the future instant at which one's aircraft will collide with an out-of-cockpit object sufficiently ahead of time to achieve evasive action is important in both fixed-wing and helicopter aviation. In the real world, as a textured terrain feature (e.g., a hillock) approaches, smaller and smaller texture elements become visible, texture elements of any given size grow larger, and the edges of sharp-edged texture elements grow perceptually sharper. Even the most technically advanced flight simulators are limited in their ability to simulate this progression. Our present findings raise the possibility that 
training with a visual display in which the dynamics of object size and texture element size are not correctly matched might increase the possibility of misjudging time to collision in a real aircraft. For example, as already mentioned, a $10 \%$ mismatch in Figure 2 produced an error of $178 \mathrm{msec}$ in a nominal time to collision of $2,000 \mathrm{msec}$, and this corresponds to a substantial $11 \mathrm{~m}$ in distance for a helicopter flying at low level at a speed of 120 knots $(62 \mathrm{~m} / \mathrm{sec}){ }^{4}$

\section{REFERENCES}

BeverLey, K. I., \& REGAN, D. (1979). Separable aftereffects of changing-size and motion-in-depth: Different neural mechanisms? Vision Research, 19, 727-732.

BEVERLEY, K. I., \& REGAN, D. (1980). Visual sensitivity to the shape and size of a moving object: Implications for models of object perception. Perception, 9, 151-160.

Beverley, K. I., \& Regan, D. (1983). Texture changes versus size changes as stimuli for motion in depth. Vision Research, 23, 1387 1400 .

Bootsma, R. J., \& VAN Wieringen, P. C. W. (1990). Timing an attacking forehand drive in table tennis. Journal of Experimental Psychology: Human Perception \& Performance, 16, 21-29.

Cavallo, V., \& Laurent M. (1988). Visual information and skill level in time-to-collision estimation. Perception, 17, 623-632.

GraY, R., \& RegaN, D. (1996a). Estimating time to collision based on binocular and monocular visual information. Investigative Ophthatmology \& Visual Science, 37, 5652.

GRAY, R., \& REGAN, D. (1996b). Estimating time to collision using binocular retinal image information alone, monocular retinal image information alone and a combination of the two. Manuscript submitted for publication.

HoYle, F. (1957). The black cloud (pp. 26-27). London: Penguin.

Karnavas, W. J., BahILL, A. T., \& REGan, D. (1990). Sensitivity analysis of a model for the rising fastball and breaking curveball. In Proceedings of the IEEE International Conference on Systems, Man \& Cybernetics. New York: IEEE.

KRUK, R., \& REGAN, D. (1983). Visual test results compared with flying performance in telemetry-tracked aircraft. Aviation. Space \& Environmental Medicine, 54, 906-911.

KrUK, R., \& REGAN, D. (1996). Collision avoidance: A helicopter simulator study. Aviation, Space \& Environmental Medicine, 67, 111-114.

LEE, D. N. (1976). A theory of visual control of braking based on information about time-to-collision. Perception, 5, 437-459.

LEE, D. N., \& LishmaN, J. R. (1977). Visual control of locomotion. Scandinavian Journal of Psychology, 18, 224-230.

Lee, D. N., Lishman, J. R., \& Thomson, J. A. (1982). Visual regulation of gait in long jumping. Journal of Experimental Psychology: Human Perception \& Performance, 8, 448-459.

Lee, D. N., Young, D. S., Reddish, D. E., Lough, S., \& Clayton, T. M. H. (1983). Visual timing in hitting an accelerating ball. Quarterly Journal of Experimental Psychology, 35A, 333-346.

LEVITT, H. (1971). Transformed up-down methods in psychoacoustics. Journal of the Acoustical Society of America, 49, 65-69.

McBeath, M. K., Shaffer, D. M., \& Kaiser, M. K. (1995). How baseball outfielders determine where to run to catch fly balls. Science, 268, 569-573.

MCLEoD, P., \& Dienes, Z. (1993). Running to catch a ball. Nature, $362,23$.

Peper, L., Bootsma, R. J., Mestre, D. R., \& Bakker, F. C. (1994). Catching balls: How to get the hand to the right place at the right time. Journal of Experimental Psychology: Human Perception \& Performance, 20, 591-612.

REGAN, D. (1991). Specific tests and specific blindness: Keys, locks and parallel processing. Optometry \& Visual Science, 68, 489-512.

REGAN, D. (1992). Visual judgements and misjudgments in cricket, and the art of flight. Perception, 21, 91-115.
REGAN, D. (in press). Visual factors in hitting and catching. Journal of Sports Science.

REGAN, D., \& BEVERLEY, K. I. (1978). Looming detectors in the human visual pathway. Vision Research, 18, 415-421.

REgan, D., \& BeverLey, K. I. (1980). Visual responses to changing size and to sideways motion for different directions of motion in depth: Linearization of visual responses. Journal of the Optical Society of America, 11, 1289-1296.

Regan, D., Beverley, K. I., \& Cynader, M. (1979). The visual perception of motion in depth. Scientific American, 241, 136-151.

Regan, D., \& Hamstra, S. (1993). Dissociation of discrimination thresholds for time to contact and for rate of angular expansion. $\mathrm{Vi}$ sion Research, 33, 447-462.

Regan, D., \& KaUShal, S. (1994). Monocular judgement of the direction of motion in depth. Vision Research, 34, 163-177.

REGAN, D., \& VINCENT, A. (1995). Visual processing of looming and time to contact throughout the visual field. Vision Research, 35, 1845-1857.

RosCoE, S. (1980). Aviation psychology. Ames: Iowa State University Press.

Savelsbergh, G. J. P., Whiting, H. T. A., \& Bootsma, R. J. (1991). Grasping Tau. Journal of Experimental Psychology: Human Perception \& Performance, 17, 315-322.

SCHIFF, W., \& DeTwILER, M. L. (1979). Information judged in impending collision. Perception, 8, 647-658.

ToDD, J. T. (1981). Visual information about moving objects. Journal of Experimental Psychology, 7, 795-810.

WARREN, W. H., YouNG, D. S., \& LEE, D. N. (1986). Visual control of step length during running over irreguiar terrain. Journal of Experimental Psychology: Human Perception \& Performance, 12, 259-266.

\section{NOTES}

1. Equation 1 holds for a rigid sphere moving at constant speed along a straight line passing directly through the observer's eye. A slightly different equation applies when the sphere is moving at constant speed along a straight line passing wide of the observer's eye (Regan \& Kaushal, 1994).

2. The effectiveness of training or transfer of training refers to the transfer to Situation 2 of a skilled behavior that has been learned in Situation 1. Roscoe (1980) developed the transfer effectiveness ratio (TER), which is used to quantify transfer of training. Transfer of training is quantified by the TER:

$$
\mathrm{TER}=\frac{\left(A-A_{s}\right)}{S},
$$

where $A$ is the aircraft training time without the use of a simulator, $A_{s}$ is the aircraft training time when a simulator is used, and $S$ is the simulator training time.

3. The case of $R=0.0$ (Figure IC) represents a crude attempt to simulate an approaching object in which the experimenter has used static texture. It is equivalent to an extended textured surface at constant distance that is viewed through a rigid square window that is approaching the eye at constant speed. Figure $1 \mathrm{~A}$ can be regarded as simulating an extended rigid textured surface that is approaching the eye at constant speed, but is viewed through the rigid square window that is also approaching the eye at a constant speed, and will arrive later than the textured surface. Figure IB faithfully simulates a rigid textured object approaching at constant speed.

4. Although our stimulus accurately simulated the retinal image of an approaching object whose motion in depth was created by any arbitrary combination of object motion and self-motion, it did not contain the radially expanding flow pattern in the extrafoveal visual field that is present in flight simulator displays. This leaves open the possibility that our findings may not generalize completely to a wide-field flight simulator display that includes a radially expanding visual stimulus for vection.

(Manuscript received September 18, 1995; revision accepted for publication February 22, 1996.) 